

\title{
Trabajadores Sociales en Israel: protagonistas y testigos del cambio social en la población árabe. Análisis de grupos de discusión y entrevistas a profesionales de los servicios sociales en Galilea y Haifa Social Workers in Israel: protagonists and witnesses of social change in the Arab population. Analysis of focus groups and interviews with professionals in the personal social services in Galilee and Haifa
}

\author{
Alfredo Hidalgo Lavié*, Javier Simonovich"* , Mirtha Ben-Nun ${ }^{* * *}$ \\ * Departamento de Trabajo Social, UNED. ahidalgo@der.uned.es, ${ }^{* *}$ Senior Lecturer. The Max Stern Academic College \\ of Emek Yezreel. javiers@yvc.ac.il, *** Social Worker. Pediatric Rehabilitation Department. Sheba Medical Center. \\ mirthaben@gmail.com
}

\begin{abstract}
Abstrac:
Qualitative Social Research Techniques applied to the production of scientific knowledge have undergone a remarkable boom in implementing research projects on topics related to our field. From its methodological strategy, this study, conducted in collaboration with The Max Stern Yezreel Valley College in Israel, aims (1) to gain insight into Social Workers perception about the central role that personal social services and social policy programs linked to them, and also focus on direct Social Work intervention with individuals, families and groups in the northern area of the country, (2) and impact the Arab minority in the processes of social change within the Israeli modern and high technological society.
\end{abstract}

Keywords: Social Work. Personal Social Services. Ethnic and religious minorities. Social Work Intervention. Qualitative Research Methodology. Israel.

\section{Resumen:}

Las técnicas cualitativas de investigación social aplicadas a la producción del conocimiento científico para la disciplina del Trabajo Social han experimentado un notable auge a la hora de implementar diseños de investigación sobre temáticas relacionadas con nuestra materia. Este estudio, realizado en colaboración con The Max Stern Yezreel Valley College en Israel, persigue como finalidad, desde su estrategia metodológica, conocer la percepción que los Trabajadores Sociales de la zona norte del país tienen (1) acerca del papel central que los servicios sociales y los programas de política social vinculados a 
ellos, y desde el enfoque también de la intervención directa en sujetos, familias y grupos, e infieren (2) en la minoría árabe en los procesos de cambio social en el seno de una sociedad moderna y de un elevado nivel tecnológico como la israelí.

Palabras clave: Trabajo Social. Servicios Sociales. Minorías étnicas y religiosas. Intervención profesional. Metodología cualitativa. Israel.

\section{Article info:}

Received: 19/10/2015 / Received in revised form: 15/01/2016

Accepted: 25/01/2016 / Published online: 30/01/2016

DOI: http://dx.doi.org/10.5944/comunitania.11.5

\section{Introducción ${ }^{1}$}

Uno de los rasgos más sobresalientes de la sociedad israelí es su diversidad social, así como toda una serie de contrastes en todos los niveles, que desde un punto de vista específicamente socio-económico, sin embargo, presenta claras contradicciones. Siendo un país referente a nivel mundial como Start-up Nation, por sus indicadores de producción económica, desarrollo tecnológico, creación científica y en materia de innovación ${ }^{2}$, uno de sus talones de Aquiles, según reza en el propio informe anual 2011 del Instituto Nacional de Seguridad Social, es "...la persistencia excepcional de la pobreza y de la desigualdad..." Unos niveles de pobreza y de desigualdad que han venido siendo muy elevados durante más de una década, incluso antes de la irrupción de la crisis económica y financiera mundial de los ochenta. Daniel Gottlieb, Director General de Investigación y de Planificación de la mencionada Agencia Nacional, afirma: "en los últimos años se han dado pasos importantes en la dirección adecuada, tales como la ley nacional de pensiones, reformas en el mercado laboral... a lo largo del país. Pero esto no parece ser suficiente para alcanzar una mejora significativa y estable... Uno de los grandes desafíos es mejorar las condiciones salariales de la población activa con menores ingresos (entre otros)"13. En 2011 , como casi en años anteriores, el 19,9\% de las unidades familiares en Israel se encuentran por debajo del umbral de pobreza, lo que se traduce en términos abso-

\footnotetext{
1 Todas las citas directas en español son de los autores de este artículo.

2 La economía israelí durante los años 2006-2012, en plena efervescencia de la crisis económica mundial mantuvo un elevado crecimiento en términos de PIB del 4,4\% anual frente al reducido 0,3\% de la zona euro. Según las estimaciones de la OCDE, para el año 2015, la economía israelí verá reducido su porcentaje al 3,5\%, pero aun así, las estimaciones de la zona euro serán sólo del 1,6\%. Research Department Staff Forecast 2013, Office of the Spokesperson and Economic Information, Bank of Israel, $y$ http://stats.oecd.org/index.aspx?queryid=9185. Euro Data Area. Última visita 21 de junio de 2014.

3 National Insurance Institute (2012). Poverty and Social Gaps, 2011. Annual Report. Jerusalem: Research and Planning Administration.
} 
lutos en 442.200 familias, que representan a 1.838 .600 personas, de las cuales 860.900 son menores. La incidencia de la pobreza en Israel representa casi el doble de la media de los países de la OCDE $(11,1 \%)$ y tres o cuatro veces superior a la de los países europeos. Un año después, en 2012, este porcentaje descendió moderadamente hasta el $19,4 \%{ }^{4}$. Un dato importante más a tener en consideración es que este porcentaje y estos números absolutos son el resultado estadístico tras considerar los beneficios y ventajas, transferencias y prestaciones económicas recibidas por las unidades familiares y los sujetos individuales; pues sin todo este paquete de medidas sociales el porcentaje ascendería al $32,8 \%$, lo que en términos absolutos se traduciría en 728.000 unidades familiares, que representan a 2.499 .100 personas, de entre ellas 1.014 .600 niños. Sin embargo, y a pesar de este público esfuerzo económico, el gasto neto en prestaciones económicas de carácter social, en relación al PIB, fue en 2013 del 15,8\%, muy inferior al $21,9 \%$ de media en los países de la OCDE y casi la mitad de lo invertido en países como Francia, Dinamarca, Bélgica, Finlandia y Suecia ${ }^{5}$.

Dos de los grupos sociales que más absorben este elevado porcentaje de pobreza son los judíos ultra-ortodoxos y los árabes (en su generalidad), cuyas unidades familiares poseen un mínimo de 6 o más miembros. Según los datos oficiales, en $2011 / 12$, el $60,4 \%$ de los árabes y el $66,9 \%$ de los ultra-ortodoxos se encontraban por debajo del umbral de la pobreza, porcentajes que se reducían al $53,5 \%$ y al $54,3 \%$ respectivamente, tras la aplicación de las bonificaciones fiscales y prestaciones económicas diversas.

Aunque en todos los informes y bases de datos oficiales estadísticos consultados se subrayan la incidencia de este alto nivel de porcentaje de pobreza y de desigualdad -en general en todo el país debido al exceso de contrataciones a tiempo parcial, los bajos salarios y las condiciones laborales en su conjunto-, se debe subrayar, que la incidencia de la pobreza es significativamente muy superior en estos dos grupos. Este cuadro económico, aunque no se corresponde con la única causa que conduce a estos elevados niveles de pobreza, ha sido, sin duda, generador de múltiples problemáticas sociales que han desbordado la capacidad de oferta de servicios y prestaciones de los servicios sociales municipales, así como de los tiempos de los profesionales para el ejercicio de la intervención. Factores de naturaleza cultural (pautas y modelos familiares), de índole geográfica (mayor número de servicios, empresas e industrias en los distritos Central y de Tel Aviv que en el Distrito Norte y de Haifa, por ejemplo), de tipo demográfico (elevados índices de natalidad en ambos grupos sociales) conforman el conjunto de elementos de exploración explicativa a esta situación dramática. No debiéndose olvidar tampoco la inversión pública en

\footnotetext{
${ }^{4}$ Adva Center (2013). Israel: A Social Report 2013. Tel Aviv: Information on Equality and Social Justice in Israel.

${ }^{5}$ En:StatExtracts.http://stats.oecd.org/Index.aspx?datasetcode=SOCX_AGG. National Accounts Database OECD. Última visita 14 junio de 2014.
} 
materia social de los últimos gobiernos y del actual, así como de la desigualdad distributiva en las partidas sociales y por territorios. Sin duda, el costoso coste por la seguridad nacional es igualmente un elemento a tener en consideración a la hora de contemplar las prioridades presupuestarias.

Este estudio se focaliza únicamente en la población árabe del distrito norte y de Haifa y persigue como finalidad analizar, desde una perspectiva de los servicios sociales (personales) en general, y del Trabajo Social, en particular, cómo el desarrollo económico, sus desequilibrios, la implementación de las políticas sociales, las transformaciones de los procesos culturales de modernización, el desarrollo tecnológico, la incidencia del conflicto político también, han afectado y de qué modo a la población árabe. Y, muy particularmente, cómo los profesionales del Trabajo Social, sensores del sistema de bienestar social, perciben estos cambios y desafíos.

La selección de todas estas variables en su conjunto responde a los siguientes criterios:

1. el mosaico étnico y religioso de la zona es mucho más diverso que en el resto de los demás distritos ${ }^{6} \mathrm{y}$, por consiguiente, el más plural en Israel.

2. Mientras que en el Distrito Centro, el $90 \%$ de la población es judía, en el Distrito Norte este porcentaje se reduce al 55\%, el cual convive con un $34 \%$ de árabes musulmanes y cristianos y un $7 \%$ de drusos. El resto de la población la componen beduinos y circasianos, entre otras minorías ${ }^{7}$.

3. En Israel, los servicios sociales son dispensados por las administraciones locales a través de sus respectivos departamentos de bienestar social. En 2010, el Ministerio de Asuntos Sociales y Servicios Sociales inició un nuevo programa de reformas encaminado a analizar las desigualdades territoriales y políticas en materia presupuestaria (Lavié, 2008).

4. Estas desigualdades entre localidades judías, árabes y mixtas y, por ende, las diferencias en la distribución de recursos por habitantes, junto con otros fac-

${ }^{6}$ La Oficina Central de Estadísticas distingue 7 distritos geográficos: Jerusalén, Tel Aviv, Haifa, Judea y Samaria, Distrito Norte, Distrito Central y Distrito Sur.

7 Se calcula que, aproximadamente el $7 \%$ de drusos equivalen a casi 120.000 personas. Este grupo social es étnica y religiosamente diferente de la población árabe, así como los circasianos, musulmanes con lengua propia que emigraron del Cáucaso y que representan unas 4000 personas, y también los beduinos, que si bien son musulmanes están integrados en la sociedad israelí y se distinguen como tales, aunque éstos, en su gran mayoría, se concentran en el distrito sur (Golub, 2009). Estas diferencias de tipo religioso y étnico también se reproducen en ciertos aspectos culturales y políticos. Por ejemplo, a diferencia de los israelíes árabes musulmanes; los beduinos, los circasianos y los drusos sirven en el servicio militar voluntariamente. 
tores de organización y planificación, así como elementos culturales y modelos familiares tradicionales de los usuarios que dificultan la intervención profesional, son los temas claves en los que se centra este estudio.

5. Las diferencias y semejanzas de los profesionales entre árabes, no árabes y judíos en cuanto a sus percepciones sobre el análisis de la situación, así como entre los propios árabes, dada su heterogeneidad, es el núcleo de esta investigación, razón que justifica la elección del Distrito Norte y de Haifa, dado su particular crisol de grupos sociales diferenciados.

\section{Consideraciones metodológicas}

Tras varios meses de análisis estadísticos de fuentes oficiales y de documentación y estudio de datos procedentes de las diferentes Agencias Públicas y delTercer Sector en materia de servicios sociales, este equipo de investigación decidió que para la realización de este proyecto se realizaran 4 grupos de discusión y un total de 12 entrevistas. Conseguida la preceptiva autorización expedida por el Ministerio de Asuntos Sociales y Servicios Sociales, se estimó en consideración la utilización de la figura del "contactador", que para algunos autores garantiza que, al evitar que los miembros de los grupos conozcan personalmente al moderador previamente, se garantiza un reclutamiento objetivo de los componentes (Orti, 1989).

Como es sabido, los grupos de discusión constituyen una técnica de recogida de datos de naturaleza cualitativa que ha sido ampliamente utilizada en diferentes campos de la investigación sociológica y cuyas posibilidades para la disciplina del Trabajo Social (Roldán, E. 1998) abren un horizonte nuevo para la obtención de hallazgos sumamente relevantes de la mano de los profesionales de la intervención directa que, con menos frecuencia de la deseada, se deciden a exponer sus conocimientos e impresiones propias de la experiencia profesional cotidiana. Su sabiduría de la praxis de la profesión, mucho menos de lo que se desearía, no es trasmitida por ellos mismos a través de artículos y libros de producción científica. Con esta finalidad, se organizaron estos grupos de discusión sobre distintos ámbitos de aplicación: 1.- la intervención profesional, para analizar las opiniones del colectivo de profesionales en torno al desarrollo y la calidad de los servicios ofertados y 2.- la evaluación de programas, desde los cuales identificar necesidades sociales, valorar su eficacia y, en suma, producir conocimiento sobre esta temática particular y sobre la población elegida. Un conocimiento que los grupos de discusión permiten aprehender, dado que "los miembros...se influyen mutuamente, puesto que responden a las ideas y comentarios que surgen de la discusión" (Krueger, 1991:24). La determinación de realizar 4 grupos de discusión, y no un mayor número, responde a la homogeneidad de los componentes seleccionados en relación al tema: todos son trabajadores sociales y cuentan con una dilatada experiencia en el tiempo en el campo de los servicios sociales. Como sugieren 
algunos especialistas en grupos de discusión, en poblaciones homogéneas, suelen desarrollarse discusiones de grupos hasta que los resultados indiquen al investigador que un grupo adicional no añadiría suficiente información nueva, lo cual suele ocurrir tras el tercer o cuarto grupo (Cohen y Engleberg, 1989, entre otros) ${ }^{8}$. No obstante, y a pesar que un primer factor es la homogeneidad en relación al objeto de estudio (Morgan, 1988) -como ya ha sido señalado- también ha sido imprescindible, dado el campo de la investigación, considerar las diferencias entre ellos mismos al pertenecer a señalados subgrupos sociales (étnicos y religiosos) y los diferentes segmentos de población sobre los que ha versado el análisis de los discursos (Lederman, 1990), a saber, la gran diversidad de la propia población árabe en la zona: cristianos, musulmanes, drusos y beduinos. La necesidad de inclusión de esta homogeneidad diversa nos ha permitido acceder al análisis de los diferentes discursos.

Respecto al reclutamiento de los miembros también se ha tenido en consideración la identidad apropiada para cada grupo de discusión para su selección, cuyo número ha permitido constituir grupos de tamaños entre 6 y 9 componentes, siguiendo las orientaciones de Morgan. Con este fin, se organizaron:

- Dos grupos mixtos de trabajadores sociales judíos y árabes (cristianos y musulmanes).

- Un grupo de discusión compuesto exclusivamente por judíos.

- Un grupo de discusión compuesto únicamente por árabes (cristianos y musulmanes).

Estos grupos se realizaron en las ciudades de Haifa y Nazareth durante los 15 primeros días de abril de 2014, con una duración de una hora y media aproximadamente cada uno.

Si bien se consideró no incluir la figura del "observador", teniendo presente que algunos autores afirman que su presencia, aunque no intervenga, ejerce un importante efecto sobre la narración y dinámica del discurso de los componentes (Robson y Wardle, 1988), el intérprete y traductor de las conversaciones, miembro del equipo de investigación, también realizó algunas de las funciones propias como observador.

Las técnicas para estimular a los participantes fueron en su mayoría de mapping, y sólo algunas proyectivas, en las que el moderador, lejos de plantear exclusiva-

8 Otros autores consideran igualmente otros criterios adicionales, por ejemplo la disponibilidad de tiempo para la investigación. Este estudio de campo se realizó durante dos meses, lo que, ciertamente, también ha influido, aunque en menor medida, a la hora de concentrar esfuerzos sobre determinados temas claves. Estos criterios también determinan, por tanto, el número apropiado y necesario de grupos de discusión (Goldman y Mc Donald, 1987). 
mente el tema (Ibañez, 1989), formuló varias y diversas cuestiones abiertas a modo de guion de la discusión (Krueger, 1991) .

En cuanto al uso complementario de la entrevista, considerada como "un proceso comunicativo por el cual el investigador extrae una información de una persona -el informante...- Esto implica que la información ha sido experimentada y absorbida por el entrevistado y que será proporcionada con una orientación e interpretación significativas de la experiencia del entrevistado" (Alonso, 1998: 67-68), fueron realizadas un total de 12 entrevistas: 3 a Coordinadores de Programas Nacionales con una de sus sedes en el distrito norte, 3 a Directores y Subdirectores de Servicios Sociales Comunitarios, 2 a técnicos de intervención directa, 2 a Directores de centros educativos y 2 a Profesores de Trabajo Social, uno de ellos Decano de la Escuela de Trabajo Social, de la Facultad de Bienestar Social y Salud de la Universidad de Haifa. De todas ellas, 6 fueron exploratorias y 6 de desarrollo y seguimiento, de diseño estructurado 6 de ellas y semiestructurado las otras 6 .

A continuación se expone, tras la debida codificación de los datos y su agrupamiento en categorías, una exposición ordenada de los temas claves abordados, considerados los más relevantes de nuestro estudio, apoyados en citas textuales numerosas que, siguiendo las sugerencias de los especialistas en grupos de discusión, "es una práctica frecuente presentar citas directas del discurso para ilustrar los resultados. La inclusión de fragmentos del discurso añadiría vida y color al informe" (Grunig, 1990).

\section{Análisis de los grupos de discusión realizados a Trabajadores Sociales árabes musulmanes, árabes cristianos y judíos}

\subsection{Valoración de la evolución histórica de los últimos años en la red de los servicios sociales}

La mayoría de los Trabajadores Sociales consideran que se han producido avances importantes en estos últimos años. Los recursos materiales y de intervención en casos y familias se han incrementado a través de la implementación de nuevos Programas, lo que ha redundado en el bienestar social de las comunidades. Esta valoración positiva no está exenta, empero, de observaciones y matices sobre la idonei-

9 En esta misma línea de actuación, se consideró seguir las recomendaciones de Henmik, según el cual: "La fase central de discusión es donde el moderador empieza a dirigir la discusión hacia los principales temas de investigación. Mover el debate hacia las cuestiones claves puede ser difícil de gestionar, ya que los participantes pueden contribuir con muchos puntos de vista y cuestiones a la vez y el moderador debe tratar de recordar estas cosas, pero cada cosa a su vez para obtener suficiente profundidad en la discusión" (Hennink, 2007: 175). 
dad y la eficiencia en algunos casos. El siempre difícil tándem necesidades sociales emergentes versus recursos existentes pivota también en el debate crítico, por lo que, finalmente, se admite simultáneamente el esfuerzo realizado por las administraciones y las insuficiencias de medios (humanos y materiales) para garantizar una atención integral.

"Yo soy Trabajadora Social de familia... en los últimos seis o siete años, en los barrios donde yo trabajo, el número de centros especializados para jóvenes creció de dos a siete... también se creó un centro de mediación para padres e hijos en el que se recibe orientaciones de tipo familiar. También (se ha puesto en funcionamiento) un proyecto denominado 'Bereshit' (Génesis) que es para chicos de hasta 9 años de edad y se realizan visitas domiciliarias con el mismo fin. $Y$ tenemos otros proyecto para niños de edad preescolar que se llama 'Shvilim' (Surcos) y son también de intervención familiar... los servicios se han desarrollado en gran manera y han traído un mayor bienestar al municipio, pero también me ha supuesto una sobrecarga de trabajo y mayor presión porque a mí me corresponde hacer diagnósticos y el seguimiento de cada caso...en los últimos 10 años, el número de casos que tengo que tratar se ha duplicado...y esto me dificulta mi tarea profesional y siento que como directora del proyecto no puedo dar una respuesta adecuada a cada una de las familias» (Trabajadora Social árabe cristiana).

"Yo trabajo con enfermos mentales y personas con discapacidad física. En las dos poblaciones hay un gran crecimiento de los servicios sociales. Creamos últimamente, en el 2010, un centro de atención especializado... es un centro de apoyo a la familia y capacitación laboral para el usuario. También ofrecemos un paquete de prestaciones que son garantizados por Ley por el Ministerio de Salud y prestamos igualmente servicios en el área de vivienda y laboral... yo creo que ha supuesto un incremento de la calidad del servicio, un trabajo más organizado y coherente>> (Trabajadora Social árabe musulmana).

"En los últimos 15 años, los servicios sociales que se han venido desarrollando, al menos en el municipio de la ciudad de Nazareth, sí responden a las necesidades de la población. Como ciudad grande que es, con una población mayoritariamente árabe, nosotros sí brindamos una respuesta adecuada. Lo que sí puedo decir es que aún no llegamos a abarcar a toda la población que tiene necesidades sociales aquí»" (Trabajador Social árabe cristiano).

"...Soy coordinadora del programa de Voluntariado... estoy como Trabajadora Social desde hace ya 25 años... nuestro trabajo es como bailar un tango, dos paso para adelante y un paso para atrás...sin embargo, cuando empecé la ayuda era restringida a la ayuda económica e individual... considerábamos al usuario como persona sin fuerza propia, sin poder. Hoy en día en cambio es que buscamos la fuerza que tiene e intentamos empoderarle para que se ayude a sí mismo y la ayuda económica es sólo un medio para proporcionarle una base determinada para que él 
pueda después desarrollarse dentro del barrio o de la comunidad" (Trabajadora Social judía).

Estos avances, insuficientes pero no cuestionados, son también resultado de la acción reivindicativa de los propios profesionales, no sólo productos de las iniciativas gubernamentales (o de las administraciones locales mismas). La labor profesional no se ha restringido exclusivamente a la acción profesional de la intervención directa con el usuario, sino también como mediador ante las autoridades, lo que ha permitido que gracias a la exposición de las necesidades y de las demandas sociales y a la labor de consulta y de asesoramiento, las agencias de la administración pública hayan impulsado iniciativas de política social en esa dirección.

"...quiero añadir que los proyectos sociales que se han creado no cayeron de arriba como paracaídas, sino que es el resultado de un gran trabajo y de un gran esfuerzo de esos trabajadores sociales que quisieron impulsar esos servicios" (Trabajadora Social musulmana).

"yo quiero decir que el proceso que se creó fue un proceso de trabajo en el que todos los trabajadores sociales estudiamos conjuntamente las necesidades de la población. Por ejemplo, identificamos el número en aumento de niños y adolescentes que sufrían abusos sexuales... comenzamos en su momento a contactar con el Ministerio de Salud, el Ministerio de Asuntos Sociales y el Instituto de la Seguridad Social y creamos un equipo de trabajo para detectar grupos en situaciones de riesgo...llegamos a crear un nivel de concienciación a nivel comunitario sobre este tema de abusos...y se promulgó una Ley para situación de riesgos aprobada en la Kneset $^{10} »$ (Trabajador Social árabe cristiano).

La ampliación de servicios, de Programas y de Proyectos, así como el incremento del número de profesionales y de usuarios ha originado igualmente un proceso de mayor burocratización en el seno de las organizaciones a medida que éstas se han vuelto más complejas en su reordenación administrativa. Una burocracia en la gestión que es diana de crítica por los profesionales.

"Yo soy la coordinadora de familias y de la comisión de diagnóstico y tratamiento... hoy hay más servicios pero para su acceso es preciso pasar por la comisión. Por Ley del Ministerio de Asuntos Sociales, en cada una de estas comisiones debe haber un Trabajador Social... hemos iniciado un proceso de burocratización muy largo. Antes no era así, sólo era preciso un Informe Social, pero ahora se requiere rellenar formularios muy largos y muy específicos..." (Trabajadora Social árabe cristiana).

10 Parlamento unicameral del Estado de Israel. 
El modelo de servicios sociales desarrollado a lo largo de estos años, a pesar de su impronta pública, especialmente en sus inicios, ha experimentado cambios con el curso del tiempo. La expansión del Tercer Sector, un rasgo muy significativo en la sociedad israelí en todos sus niveles, también ha venido irrumpiendo con fuerza en el campo de los servicios sociales, así como las fórmulas de corresponsabilidad entre el sector público y el sector privado (lucrativo o no), siendo esto último, especialmente, motivo de discusión y de crítica por los profesionales.

"...en los últimos años, se puede ver claramente un aumento tanto de usuarios atendidos como del volumen de trabajadores sociales en plantilla...se ha producido un gran avance pero no es aún una realización real pues estamos más cerca del modelo inglés o neoliberal y menos del modelo socialdemócrata. A pesar de que los servicios que ofrecemos son muy buenos y van en aumento, paralelamente hay privatizaciones, y el copago es un impedimento para los usuarios... creándose un abismo entre lo que solicitan y lo que se les puede ofrecer» (Trabajador Social árabe musulmán).

\subsection{La intervención profesional directa en función de la pertenencia étnica o religiosa}

La cohabitación en un mismo espacio geográfico de diversos grupos sociales y considerando la pertenencia de los propios profesionales a grupos sociales específicos, ha dado lugar a un modelo de distribución de funciones y de especialización entre los técnicos. Las razones de que el profesional de referencia pertenezca al mismo grupo social, en general, responde más a cuestiones de analogías culturales y lingüísticas que a elementos políticos de segregación.

"Cuando yo comencé, nadie me dijo que usted trabaje sólo con árabes, si bien el barrio era mayoritariamente árabe, pero si me encontraba con ciudadanos de origen ruso o con etíope, pedía ayuda a los compañeros si había alguna cuestión cultural particular...y otro ejemplo es que en nuestros proyectos comunitarios trabajan conjuntamente judíos y árabes..." (Trabajadora Social árabe musulmana).

"Yo comencé también a trabajar indistintamente con todos los grupos y cuando se hace diferencias es para entender mejor y disponer de mayor sensibilidad cultural, pero no basado en ninguna cuestión de discriminación...» (Trabajadora Social árabe musulmana).

"Yo también quiero agregar que lo que resulta es que se trabaja por la base del idioma y la sensibilidad cultural, pero yo soy cristiana y trabajo con población musulmana y no hay ningún tipo de diferencia. La diferencia es más del grupo étnico y no de la religión...cuando no hay necesidad de tocar las diferencias étnicas se puede trabajar de modo general» (Trabajadora Social árabe cristiana) 
"Mi sensación es que trabajamos de acuerdo a los distintos grupos étnicos. Trabajadores sociales árabes trabajan fundamentalmente con árabes por la cuestión de la lengua y los que hablan 'amhari', que es el idioma que hablan los etíopes, la mayoría son etíopes también» (Trabajadora Social judía).

"Yo trabajo en un barrio con jóvenes en situación de riesgo. Trabajo con los que hablan árabe y si hay algún joven que hable sólo hebreo, tenemos otro profesional que habla hebreo...» (Trabajadora Social árabe cristiana).

\subsection{Problemáticas a la hora de la intervención profesional en los modelos tradicionales de familias}

Los procesos de modernización social y cultural en una sociedad avanzada como la israelí han generado problemas análogos al modelo occidental de transformación en el sistema de la familia nuclear. La relajación de las costumbres, la occidentalización en las pautas y conductas de comportamiento, el deseo de emancipación de los jóvenes, la incorporación de la mujer en el mercado laboral, más acusado lógicamente en los centros urbanos que en las zonas rurales, generan inconvenientes y reservas por parte de los usuarios a la hora de la intervención y de la mediación familiar.

"Muchos de los que vienen aquí piensan que destruimos a las familias porque les damos empoderamiento a la mujer, porque va en contra de la cultura árabe, ...porque antes los problemas se solucionaban dentro de la jamula (familia extensa o tribu en árabe), dentro de la casa, como si nosotras debilitásemos la imagen del hombre y fortificásemos la imagen de la mujer. Y nosotras llevamos a la práctica la política social del modelo israelí entre comillas frente a la sociedad árabe..." (Trabajadora Social árabe musulmana).

"Yo pienso que la diferencia principal es que cuando las mujeres árabes se salen del camino, no tienen camino de vuelta, desde el punto de vista de la jamula... además de estar en peligro de muerte... sus vidas corren peligro si llegan a acercarse de nuevo a su casa. La mayoría vienen de sociedades patriarcales tradicionales, cosas que una adolescente judía hace en su vida cotidiana, salir con un muchacho, por ejemplo, (en las chicas árabes) ya reciben el nombre de prostitutas, y el futuro de ellas ya está hipotecado... es ostracismo, dejan de pertenecer a la sociedad..." (Trabajadora Social judía).

"Yo estudio con 5 mujeres árabes, unas son Trabajadoras Sociales y otras son consejeras de educación y ellas afirman que cuando descubren, por ejemplo, agresiones, la dirección no les permite seguir trabajando el tema. Es imposible tratar el tema desde los recursos sociales dentro del pueblo... las mujeres que son tratadas son consideradas como que ya no se pueden casar y sacar a una chica de una casa 
por malos tratos no se puede hacer porque en seguida le estás dañando la reputación...» (Trabajadora Social judía).

«...la mayor parte de las familias que acuden al Departamento... el hombre está desocupado o parado o drogadicto o posee cualquier otro tipo de problema y entonces es la mujer la que tiene que salir a trabajar para mantener a la familia...y hay casos además de hombres que se casan con otras mujeres en Turquía o en Egipto y el marido se va y la mujer se queda aquí con sus hijos... y no tiene nada..." (Trabajadora Social árabe musulmana).

"La perspectiva de los usuarios y de los trabajadores sociales es distinta. Los usuarios todavía nos ven como un lugar donde se puede recibir ayuda económica, conseguir la prestación y marcharse y nuestro objetivo es que pasen por un proceso personal de empoderamiento" (Trabajadora Social árabe cristiana).

\subsection{Desigualdades y desequilibrios en la distribución presupuestaria y de recursos entre comunidades judías y árabes}

La insuficiencia de medios y de financiación no responde exclusivamente a la superación de las demandas sociales frente a los recursos sociales disponibles. Los técnicos reconocen que la existencia de estos desequilibrios se produce y está injustificado, son desequilibrios territoriales, pero a su vez, afectan directamente más a las comunidades árabes que a las pobladas mayoritariamente por judíos. La discusión sobre si la naturaleza de esta discriminación es de origen político o responde a otras causas se abre a múltiples interpretaciones con argumentos diferentes.

"Yo como ciudadano israelí árabe veo que hay diferencias en los presupuestos que se reciben en los municipios árabes... incluso en ciudades mixtas, de árabes y judíos, se pueden ver las diferencias en los servicios de bienestar social, en educación y en todos los servicios...pero también vemos un proceso nacional de disminución del Estado del Bienestar y se están reduciendo las prestaciones económicas en general y muchas familias árabes dependen de estos recursos..." (Trabajador Social árabe cristiano).

"Yo creo que el gobierno pretende mantener el Estado judío y los árabes son un problema demográfico y de lo que se trata es de limitarlo, por eso es importante no invertir en la población árabe para que no crezca. La idea es hacer menos y desarrollar menos» (Trabajadora Social árabe cristiana).

"Porque es un gobierno conquistador y un pueblo conquistado... el motivo por el cual se crea la discriminación es porque esa es la política del gobierno" (Trabajadora Social árabe musulmana). 
"En el Estado de Israel somos todos iguales en una cosa, en una característica: la discriminación la tenemos todos, en eso, todos somos iguales, porque todos nos discriminamos a todos. Pero desde el punto de vista del Estado hay una discriminación sistemática en todas las áreas, por ejemplo, en los tipos de trabajo a los que puedes acceder...» (Trabajador Social árabe musulmán).

"Yo trabajo con todos ustedes aquí desde hace muchos años y me es muy difícil entender esa sensación de discriminación que decís. Pienso que puede ser quizá cierto en algunas cosas, pero también nosotros, los judíos de Israel, que somos conocidos como 'sabras', sentimos la discriminación de todos con todos, judíos sefardíes, asquenazíes, etíopes o rusos. Yo quiero creer y así lo pienso que yo doy servicios a toda la población y tengo la convicción que aquí, en nuestro lugar de trabajo, no hay discriminación entre árabes y judíos» (Trabajadora Social judía).

«Mi sensación aquí en el Departamento es de igualdad sin duda, pero no olvidemos que los servicios sociales trabajan con población discriminada en todos los aspectos..." (Trabajadora Social judía).

"Yo quiero decir también la parte en la que nosotros, como sociedad, tenemos responsabilidad: nosotros también como comunidad tenemos la responsabilidad de cambiar la situación y yo no estoy de acuerdo en decir siempre 'el gobierno discrimina, el gobierno discrimina', a pesar de que hay discriminación gubernamental...» (Trabajadora Social árabe cristiana).

La discriminación, los desequilibrios presupuestarios, la distribución de recursos reconducen la discusión y el debate a la cuestión política. Esta problemática no está ausente tampoco en el campo de los servicios sociales ni de los profesionales. Se podría afirmar incluso que las diferentes lecturas que cada uno de ellos hace del 'conflicto' impregna también de contenidos múltiples cuando se habla sólo de servicios sociales. La discriminación, intencionada o no, políticamente hablando, es sólo el aspecto superficial bajo el cual subyace la cuestión del conflicto y en el que un análisis autocrítico de la situación no es expresado, voluntariamente, en su total libertad.

"Me es difícil referirme a una autocrítica cuando viene a preguntármelo 'un factor externo'...por ejemplo, yo puedo tener una crítica de mi sociedad de aquí hasta no sé dónde, pero si hablo ante 'un factor externo' matizo la crítica, porque yo siento que si respondo, el gobierno va a venir en seguida a culparnos a nosotros... y a veces siento también que traiciono a mi comunidad si llego a decir ciertas cosas, pero tampoco quiero tomar la posición de la sociedad árabe como víctimas porque estoy cansada también de esa posición" (Trabajadora Social árabe cristiana).

"Tampoco elegimos adecuadamente a los políticos y a las personas con responsabilidad... no hemos conseguido nosotros los árabes juntar las manos con los judí- 
os que quieren juntar sus manos para hacer cosas juntas» (Trabajador Social árabe cristiano).

"Nosotros, todos, trabajamos en bienestar social con familias segregadas en todos los aspectos de la vida. Algunas son segregadas por su situación económica, otras son segregadas por ser inmigrantes y también está claro que tenemos familias segregadas por su pertenencia étnica o cultural, pero nosotros nos encontramos con todo el espectro por todos los motivos, problemáticas y diferencias" (Trabajadora Social judía).

"Hay discriminación en varios ámbitos, por ejemplo, si comparamos una aldea árabe y una aldea judía, hay servicios que en la primera no hay y sí en la segunda, y si los hay, la calidad no es la misma. También, por ejemplo, en el web del servicio nacional hay mucha información más en hebreo que en árabe $y$, por supuesto, el tema de la accesibilidad del idioma es importante en Israel...." (Trabajadora Social árabe cristiana).

\section{Análisis de las entrevistas realizadas a los diversos profesionales del ámbito de Trabajo Social y de la Educación}

Las desigualdades y desequilibrios en la oferta de servicios sociales en los municipios de mayoría árabe frente a las localidades de mayoría judía, o ciudades mixtas, es una de las cuestiones centrales sobre las que pivota las principales quejas de los profesionales. Una realidad que la propia Administración Central ha reconocido, a través de sus estudios e informes, y sobre la que se ha comprometido, desde hace unos años, en alcanzar una solución. Estos desequilibrios, empero, no se reducen exclusivamente al campo de la distribución presupuestaria en materia de servicios sociales, sino que, como se ha señalado, afecta en muchos aspectos a un amplio espectro muy diverso de la sociedad israelí, especialmente a la población activa contratada a tiempo parcial con baja remuneración salarial, entre otros. Desigualdades y desequilibrios que se reproducen, de igual modo, entre los propios diversos grupos sociales judíos ${ }^{12}$ y también entre territorios (Distrito Centro yTel Aviv, frente a los

${ }^{11}$ A colación de este comentario sobre la accesibilidad del idioma, y aunque pueda resultar puramente anecdotario, esta intervención nos permitió posteriormente comprobar que todos los carteles del centro de servicios sociales en el que nos encontrábamos, estaban escritos en hebreo. Al preguntar al responsable del Programa por qué no estaban los anuncios escritos también en árabe, el técnico respondió lo siguiente: "Ni siquiera me di cuenta y el que escribió los carteles y los diseñó fui yo mismo y no me di cuenta. Desde luego cuando hay eventos importantes los carteles están todos en los 4 idiomas: hebreo, árabe, ruso y amhari" (Trabajador Social árabe musulmán). Esta respuesta está registrada literalmente en la base de grabaciones realizadas de todo el estudio y que puede ser comprobada.

12 Las desigualdades salariales entre árabes y judíos son sustanciales, así como entre los judíos orientales o mizrahim (procedentes de África y Asia) y los judíos asquenazíes (de Europa y América). En 2012, 
Distritos Norte y Haifa), en los que la concentración de la actividad económica, a escala nacional, es un factor explicativo tanto de los desequilibrios en las condiciones laborales como en las posibilidades de acceso al mercado laboral.

La coincidencia de los distintos puntos de vista de los diversos actores (profesionales de la intervención directa, Administraciones, Agencias del Tercer Sector, personal investigador y docente) sobre esta cuestión es prácticamente unánime. Las diferencias son, sin embargo, mayores a la hora de interpretar las razones por las cuales se producen. Si la discriminación es de naturaleza explícitamente política, e intencionada o no, forma parte del debate de discusión, en la cual subyace el discurso ideológico sobre el conflicto árabe/palestino-israelí/judío.

«Podemos afirmar que el Gobierno, oficialmente, no realiza una política directa de discriminación, pero si observamos la panorámica de la comunidad árabe, comparada con la comunidad judía en materia de salud, bienestar social, educación, vivienda y otros servicios sobre los que tiene responsabilidad el Gobierno, se aprecia una ruptura en cuanto a asignaciones y se encuentran fácilmente diferencias...Pero ahora, y desde los últimos 20 años, se aprecia que muchos líderes políticos y ministros diferentes reconocen que existe esta brecha... es un reconocimiento honesto de los gobiernos... y encontramos ahora más iniciativas lideradas por el gobierno, por ejemplo, sobre lo que debemos hacer en la educación superior...tarea que yo mismo lidero como Vicepresidente de la Comisión Nacional de Planificación y Presupuestos de la Asamblea de Educación Superior de Israel» (Prof. Faisal Azaiza, Director de la Escuela deTrabajo Social de la Universidad de Haifa).

"En mi opinión, sobre los presupuestos, de acuerdo con los puestos que debe haber, recibimos lo correcto según la fórmula legal del Ministerio de Bienestar Social. La diferencia radica en la participación local de los presupuestos del municipio $^{13}$ o en el reparto posterior, interno a nivel local, que puede no ser justo. Por eso, por parte del Ministerio de Bienestar Social se está realizando una investigación para ver cómo es la división de la carga de trabajo de los trabajadores sociales para contemplar una división más justa. Es un estudio para todo el país, no es sólo un problema de Nazareth...no hay discriminación institucional» (Ramsi Nazar, Director de zona de los servicios sociales comunitarios del municipio de Nazareth).

el promedio de los beneficios salariales obtenidos por estos últimos era de un $42 \%$ superior que los del cualquier otro trabajador de zona urbana. Diferencias que también se producen con los judíos de origen etíope. "Ashkenazim, Mizrahim and Arabs" en: Adva Center (2013). Israel: A Social Report 2013. Tel Aviv: Information on Equality and Social Justice in Israel, 14.

${ }^{13}$ Sobre este punto, el mismo entrevistado señala lo siguiente: «...depende de las posibilidades económicas del municipio, de acuerdo con lo que recaudan y las necesidades que tienen... en Nazareth son muy pequeñas, pero Haifa, por ejemplo, tiene puerto y eso genera ingresos". Los porcentajes de desempleo en estas zonas puede ilustrar, en cierto modo, esta afirmación. Mientras que en Nazareth es del 14,5\%, en Haifa es sólo del 5,0\% y en Tel Aviv-Jaffa del 3,5\%. 
"En general, cuando yo miro este tipo de situaciones lo que yo veo es una visión más estructural de las diferencias que hay en Israel y cómo estas diferencias se manejan en los sistemas de bienestar, en los servicios públicos...hay como un estado de negación muy grande de las diferencias nacionales que hay o el impacto que tiene el conflicto árabe-judío, palestino-israelí en estas relaciones. Hay como una ceguera... Dentro de ellas hay factores que tienen que ver también con la sociedad, la cultura, los modos, las normas del propio grupo social, pero también hay que verlas dentro de un contexto mucho más amplio...yo trabajo con pobreza y veo cómo el sistema, por ejemplo, crea lagunas donde pasan cosas que realmente no tendría que pasar» (Roni Strier, Profesor de Trabajo Social de la Universidad de Haifa).

El cambio social que el proceso de modernización a través de su desarrollo económico, los avances científicos y tecnológicos, la expansión de la sociedad de consumo y del ocio, ha afectado igualmente a la naturaleza de las demandas sociales y de las necesidades que llegan a las puertas de los servicios sociales. Todo un desafío para los propios profesionales a la hora de realizar intervenciones en el medio familiar y de grupo y cuya eficiencia, sin la participación y disponibilidad de los usuarios en su conjunto, dificultan su tarea.

"Hay una diferencia abismal entre la generación anterior, la de los mayores, con sus valores, y la gente joven de hoy. El abismo generacional es muy fuerte. Por ejemplo, una mujer joven que quiere conocer a alguien para formar una familia y casarse, el código tradicional de la sociedad no se lo permite e interfiere la familia para impedírselo y esto genera muchos conflictos entre los jóvenes y los padres... o conoce a alguien de una manera secreta sin ir por la vía de los códigos normales y se enfrentan a sus padres que les recrimina que su conducta está en contra de la tradición" (Trabajador Social beduino).

"Es un problema social, cultural, que tienen en la sociedad y que hay que conocer. El hombre cuando termina los estudios secundarios..., si termina porque muchos suspenden a la edad de 16 o 17 años. ¿Por qué suspenden? Por la razón de que él tiene que ganar bastante dinero para casarse, ningún padre quiere casar a su hija con alguien que no tiene casa o dinero...La razón ideológica (para las mujeres) es que ellas quieren un futuro diferente al de sus padres, ellas dicen: 'yo tengo 17 años y quiero construir mi futuro y puedo comenzarlo ahora, no quiero quedarme sentada en casa y esperar que un hombre quiera casarse conmigo y que pague todo de mi vida y yo me quede en casa con todos mis hijos" (responsable de la ONG Ha'aguda Le'hitnadvut) ${ }^{14}$.

14 La ONG Ha'aguda Le'hitnadvut es la Organización No Gubernamental más antigua, y con mayor número de voluntarios, que gestiona el conocido Sherut Leumi (Servicio Nacional Civil), alternativo al servicio militar obligatorio, prescriptivo para todos los hombres y mujeres judíos en Israel. A este Servicio se acogen principalmente mujeres que aprenden y desempeñan tareas y funciones en los servicios de 
"Muy pocas mujeres viven solas y las que están no tienen el permiso de la comunidad. Pero creo que debería haber un cambio en esto... no creo que las mujeres drusas se sientan más 'liberadas', también depende de dónde vivan, por ejemplo, si viven en la ciudad es más fácil que si viven en pueblos porque están más expuestas a la sociedad... una mujer drusa sola trabajando para una compañía aérea, por ejemplo, no es aceptada generalmente...creo que se debe a las normas familiares..." (Trabajadora Social drusa).

La pobreza, la escasez de oportunidades y la insuficiencia de recursos junto con los conflictos generacionales entre los modelos tradicionales y los procesos de occidentalización en las pautas de comportamiento, generan una serie de problemas sociales que afectan al orden público como son la delincuencia, el incremento del consumo de sustancias tóxicas, la prostitución, y lo que es aún más dramático, la violencia de género ${ }^{15}$.

«El cambio en este país va a llevar años... yo me acuerdo, hace ahora ya casi 30 años cuando comencé a ejercer como trabajadora social, y no había una Ley contra la violencia familiar, y recuerdo que se decía entonces '¿qué derecho tiene el país de intervenir en lo que sucede dentro de mi casa?'... pero hoy el problema es más complejo, de repente estás en una sociedad que te dice 'no querido, acá vos tenéis los mismos derechos de todos' y te responden '¿quién te da permiso a vos a venir con esos derechos y esos valores a obligarme a mí a recibirlos?'...(para ellos) cualquier cambio va a ser más occidental, lo que quiere decir más sionista, y esto es señal que le están derrotando... lo que yo veo es que en Israel la mayor parte de la población es laica, más moderna, más occidental, pero tienes dentro de la misma sociedad, sociedades más tradicionales, que pueden ser de los judíos religiosos y de los árabes que son mucho más tradicionales. Las mujeres están en estos mundos como hace 100 años en España... y hoy día, puedes tener al lado de tu casa a una mujer que no la dejan salir con el novio, no me refiero a tener relaciones sexuales con él, sino a no salir con él a solas..." (Beatriz Rozen-Katz, Coordinadora del Programa Nacional OFEKNASHI ${ }^{16}$ en Haifa).

bienestar social, especialmente. En los últimos años, el número de mujeres árabes cristianas que se suman a este a Programa no cesa de incrementarse.

${ }^{15}$ Según el Informe elaborado por Public Security Ministry en 2012, bajo el título “Women in the Crosshairs", del 2003 al 2011 se ha producido un incremento alarmante de muertes por violencia de género. La proporción de mujeres musulmanas y drusas asesinadas es más del doble que la proporción de las mujeres judías. La Division for the Status of Women at WIZO Israel, considera que estas cifras no son exactas porque no incluyen el elevado número de mujeres árabes desaparecidas.

${ }^{16}$ EI Programa OFEKNASHI es un Programa Nacional por iniciativa del Ministerio de Asuntos Sociales y Servicios Sociales hace más de cinco años cuya finalidad consiste en asistir a las mujeres que se dedican a la prostitución o proceden de ella, incluyendo casos de violencia de género y drogadicción. Además del equipamientoque disponen en Haifa, el Programa cuenta con otros 3 centros distribuidos en diferentes zonas del país. 
"Es un mundo en el cual yo no puedo estar. Me despierta cosas del pasado y yo no las quiero en mi vida. Con mi familia, hace años que no tengo relación. Es uno de los precios que yo pago por ser árabe, que en nuestro caso la familia no perdona... Yo nunca me sentí culpable de ser lesbiana. Era lo único que tenía claro, pero el precio más alto que pagué fue la drogadicción. Es un mundo que da mucha fuerza a los hombres. El padre tiene la primera y la última palabra. Es un mundo que deprime las necesidades nuestras como mujeres. Nuestra misión es traer hijos, cocinar... yo pagué el precio con la soledad, con no pertenecer a nadie, no vivo en mi sociedad y tampoco pertenezco a la sociedad judía" (monitora árabe cristiana del Programa Nacional OFEKNASHI en Haifa).

\section{Conclusiones}

1. Los Servicios Sociales (personales) han experimentado, en los últimos 20 años, un notable avance, tanto en el incremento de recursos humanos y materiales, como en la implementación de nuevos Programas y Proyectos de políticas sociales sectoriales.

2. La naturaleza de las prestaciones y de los servicios, igualmente, han experimentado un cambio muy importante en su transformación del modelo de acción social asistencial de sus inicios a la intervención profesional integral y global con el usuario. Todo un proceso de profesionalización y modernización de la acción social al más puro estilo occidental.

3. Estos avances notables en el campo de los Servicios Sociales son resultados, no sólo de la voluntad política del legislador y de la Administración central y local, sino también del asesoramiento y de la reivindicación protagonizada por los propios profesionales, tanto al frente de los servicios públicos como de los servicios del Tercer Sector.

4. Pero esta expansión del sector público y del sector privado también ha impuesto su propia dinámica en los procesos de burocratización de la gestión de servicios, motivo de queja de los profesionales que ven obstaculizadas sus tareas cotidianas a la hora de intervenir en casos y familias.

5. Los recursos sociales existentes son, empero, insuficientes. La emergencia de nuevas necesidades sociales y el desarrollo significativo de la demanda dificultan la eficiencia de la intervención de los profesionales, tanto en los tiempos requeridos para la atención, como para los posteriores procedimientos de seguimientos de los casos.

6. Al igual que en los modelos occidentales de sistemas de bienestar social, y de las políticas gubernamentales a la hora de su implementación de programas y 
proyectos, los servicios públicos (tanto en su titularidad como en su gestión) han ido cediendo espacio a otros actores y agentes sociales, dado lugar a formas de privatización duramente criticada también por los trabajadores sociales israelíes, tales como la expansión de fórmulas jurídicas más económicas que el clásico intervencionismo público (como las subvenciones, conciertos, sistemas de copagos, etc.).

7. Una de las particularidades del modelo israelí es el nivel de elementos diversos que dan a lugar a su peculiar crisol cultural de sus habitantes (y de sus profesionales también). La diversidad lingüística, étnica y religiosa, y todos estos años de convivencia en una admirable armonía -considerando la complejidad del contexto político-histórico-, tanto entre los propios trabajadores sociales como entre los usuarios, ha impuesto una razonable dinámica distributiva. Lejos de cualquier intencionalidad política de segregación racial, los profesionales se consagran a usuarios y comunidades, en función de criterios de racionalidad: conocimiento de la lengua, de las costumbres y usos y de las pautas de conducta y de comportamientos; considerando esta asignación un elemento de garantía para una intervención profesional más eficaz y eficiente.

8. Este crisol cultural, enriquecedor para una sociedad abierta como la israelí, no está exento de problemáticas, como acontece igualmente en otros modelos nacionales de amplia heterogeneidad cultural. La concepción tradicional del modelo de familia, de las relaciones sociales, de la distribución de roles y de pautas culturales patriarcales suponen un claro desafío para los profesionales a la hora de la intervención profesional, considerada esta intervención, para estos grupos y comunidades, un 'intrusismo' en sus vidas privadas y una 'injerencia' al estilo occidental de resolver los problemas internos de cada grupo y cuestiones domésticas de cada jamula o clan.

9. Las (ciertas) desigualdades y desequilibrios presupuestarios en forma de inversión pública entre municipios, de mayoría judía o árabe, o mixtas, eleva el debate a la cuestión política. A la interpretación sobre la naturaleza de su intencionalidad. A la existencia, o no, de discriminación institucional explícita. Y sobre esta cuestión particular, se aprecia que no hay una sola y única respuesta unánime. Se ha observado, tras el análisis, que dentro de un mismo grupo social coexisten percepciones no compartidas, lo cual es producto, una vez más, de la gran heterogeneidad social del modelo israelí, que lejos de encasillar percepciones y apreciaciones homogéneas por grupo, la libre interpretación y el análisis es transversal y no condicionada, en su totalidad, por la pertenencia al grupo.

10. Un claro ejemplo a este respecto, y a modo de conclusión final, es que la discriminación ni es unidimensional ni posee una exclusiva raíz política. También las formas y estilos de vida producen pautas discriminatorias en el seno de los 
propios grupos y comunidades. Y la segregación resultante no posee un único frente entre mayoría y minoría, sino también entre las mismas minorías y en el seno propio de ellas mismas. Esto puede explicar que los trabajadores sociales israelíes, independientemente de su condición étnica, religiosa y lingüística, trasciendan la conflictividad, siempre subyacente, del conflicto político, a los términos propios, y de definición, del conflicto social, producto de la diversidad, característico del modelo occidental. $Y$ esta interpretación posee un fundamento empírico: su dilatada experiencia profesional al frente de los Servicios Sociales desde los cuales se aborda la discriminación en todas sus múltiples facetas.

\section{Referencias}

Alonso, L.E. 1998. La mirada cualitativa en Sociología. Madrid: Fundamentos.

Azaiza, F. y Brodsky, J. 2003. The Aging of Israel's Arab Population: Needs, Existing Responses, and Dilemmas in the development of services for a society in transition. IMAJ, vol.5, 383-386.

Bligh, A. 2005. The Israeli Palestinians: An Arab Minority in the Jewish State. Oregon: Frank Cass Publishers.

Cnaan, R. A. 1998. Social Services for the enemy? Education for Social Work and the Arab sector in Israel, International Social Work, Vol.31, 33-43.

Cohen, M. C. y Engleberg, I.N. 1989. Focus group research: procedures and pitfalls. Comunicación presentada en la Convention of the Eastern Communication Association. Ocean City, MD. ERIC Document Reproduction Service, $\mathrm{n}^{\circ}$ ED307001.

Eisenberg, L.Z., Caplan, N., Sokolof, N.B. y Abu-Nimer, M. 2003. Traditions and Trasintion in Israel Studies, Volume VI, Albany: State University of New York Press.

Eisikovits, Z., Griffel, A., Grinstein, M. y Azaiza, F. 2008. Attitudes of Israeli Arab Social Workers concerning Woman Battering, The Relatioship between Responsibility, Societal Reaction and Legitimization of Violence. Journal of Social Service Research, Volume 26, Issue 3.

Fass, H. y Cohen-Navot, M. 2012. Work with Families by Social Services: Outcomes, Intervention Strategies, and the Role of Professional Social Workers. Jerusalem: MyersJDC Brookdale Institute (Engelberg Center for Children and Youth) and Research, Planning and Training Division (Ministry of Social Affairs and Social Services).

Goldamn, A. E. y MC Donald, S.S. 1987. The group depth interview. Principles and practice. New Yersey: Prentice Hall Inc.

Golub, J.E. 2009. The Galilee, Israel. Self-Evaluation Report. OECD Reviews of Higher Education in Regional and City Development. Jerusalem: The Council for Higher Education of the State of Israel.

Grunig, L.A. 1990. Using focus groups research in public relations. Public Relations Review, XVI (2), 36-49.

Hennink, M. 2007. International Focus Group Research. A Handbook for the Health and Social Sciences. Cambridge: Cambridge University Press. 
Ibáñez, J. 1989. Cómo se realiza una investigación mediante grupos de discusión. Pp. 489-501, en García Fernando, M; Ibáñez, J y Alvira, F. El análisis de la realidad social, Madrid: Alianza Editorial.

Krueger, R. A. 1991. El grupo de discusión. Guía práctica para la investigación aplicada. Madrid: Pirámide.

Lavié, Y. 2008. Family Policy in Israel. Jerusalem: Research, Planning and Training Division, Ministry of Social Affairs and Social Services.

Lederman, L. C. 1990. Assesing Educational efectiveness: the focus group interview as a technique for data collection. Communication Education, 38, 117-127.

Morgan, D. L. 1988. Focus groups as qualitive research. Sage University Paper Series in Qualitive Research Methods. Beverly Hills: Sage Publication.

Ofek, A. 2009. Report of the Social Service Reform Committee. Jerusalem: Ministry of Social Affairs and Social Services.

Orti, A. 1989. La apertura y el enfoque cualitativo o estructural: la entrevista abierta y la discusión de grupo. En García Fernando, I. y Alvira, F. El análisis de la realidad social. Métodos y técnicas de investigación. Madrid: Alianza.

Robson, S. y Wardle, J. 1988. Who's watching whom? Journal of The Market Research Society Conference, 30 (3), 191-233.

Roldán, E. 1998. Los "Grupos de discusión" en la investigación en Trabajo Social y Servicios Sociales. Cuadernos de Trabajo Social, 11, 133-144. 


\section{comunitania}

REVISTA INTERNACIONAL DE TRABAJO SOCIAL Y CIENCIAS SOCIALES

INTERNATIONAL JOURNAL OF SOCIAL WORK AND SOCIAL SCIENCES

\section{ARTICULOS/ARTIILLS}

El arte urbano como instrumento de empoderamiento y visibilización. El Festival de Asalto/

Urban art as an instrument of empowerment and visibility. Asalto festival

Cecilia Serrano-Martínez.

Págs 9-26

¿Usuarios o ciudadanos? Intervención y participación en las políticas y servicios sociales

/ Users or citizens? Intervention and participation in social politics and services

Miguel Arenas Martínez.

Págs 27-43

La violencia de género en las historias de vida de las mujeres que inician su trayectoria vital en la situación de vulnerabilidad / Gender-based violence in the life stories of women who begin their life trajectories in situations of vulnerability

Gabriela Moriana Mateo

Págs 45-67

Las consecuencias de la burocratización en las organizaciones de servicios sociales / The consequences of bureaucratizacion in the social service organizations

Xavier Montagud Mayor.

Págs 69-89

Trabajadores Sociales en Israel: protagonistas y testigos del cambio social en la población árabe Análisis de grupos de discusión y entrevistas a profesionales de los servicios sociales en Galilea y Haifa / Social Workers in Israel: protagonists and witnesses of social change in the Arab population. Analysis of focus groups and interviews with professionals in the personal social services in Galilee and Haifa

Alfredo Hidalgo Lavié, Javier Simonovich y Mirtha Ben-Nun

Págs 91-111

Software educativo Historits 1.0: Una propuesta innovadora para enseñar y aprender la historia del Trabajo Social / Educational software Historits 1.0: an innovative offer to teach and to learn the history of the Social Work

Eloy Casique Rojas y Yocelyn Castro.

Págs 113-139

"Lauream Pacis": Una Cultura de Paz a través del deporte / "Lauream Pacis": Peace Culture trough the Sports

Paris Alejandro Cabello Tijerina y Lina Guadalupe Sierra García

Págs 141-155

Medicina indígena tradicional, su enlace con la psiquiatría y la salud Pública / Traditional native medicine, its linkage with psychiatry and public health

Iván Noé Martínez-Salazar, Erika Acevedo-Stefanoni y Luis Angel Martínez-Hernández.

Págs 157-168

\section{BESEEIAAS/REVIEWS}

Antonio López Peláez. Teoría del Trabajo Social con Grupos. Segunda Edición revisada y ampliada /Theory of Social Work with Groups. 2 ed.

(por Emilio Díaz de Mera).....

Págs 169-174

Roberta Teresa Di Rosa. Mediación, Ciudadanía y Convivencia entre Culturas / Mediation,

Citizenship and Coexistence among Cultures

(por Alberto José Olalde Altarejos).

Págs 175-177

Maria Antonietta Selvaggio (a cura di), Educatrici di società. Racconti di donne e di cura / Educadores de la sociedad. Relatos de mujeres y del cuidado 2014

(por Fiorenza Deriu)

Págs 179-182

Enrique Pastor Seller. Trabajo Social con Comunidades / Social work with communities 2015 (por José Javier Navarro Pérez).

Págs 183-185

Enrique Pastor Seller, Gerardo Támez González y Karla Annett Cynthia Sáenz López.

Gobernabilidad, ciudadanía y democracia participativa. Análisis comparado España México /

Governance, citizenship and participatory democracy 2014. A comparative analysis between

Spain and Mexico. Madrid: Dykinson

(por Juan Bautista Martínez Fernández)

Págs 187-192

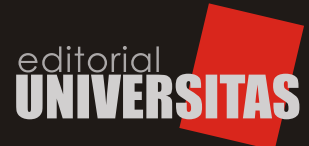

